\title{
ESTRATEGIAS PARA REDUCIR LA DISLALIA EN NIÑOS DE NIVEL INICIAL
}

\author{
STRATEGIES TO REDUCE DISLALIA IN CHILDREN OF INITIAL LEVEL
}

\section{Cecilia Guevara ${ }^{\circledR}$, María Noboa, Jair Vistín, Araceli Lucio, Geofre Pinos.}

Universidad Estatal de Bolivar, Campus Académico "Alpachaca” Av. Ernesto Che Guevara s/n y Av. Gabriel Secaira. Guaranda - Ecuador.

Email:gsilvana45@hotmail.com

https://doi.org/10.33789/talentos.7.2.136

\begin{abstract}
Resumen: El objetivo de la investigación fue determinar la estrategia que contribuya a reducir la dislalia, describiendo los fundamentos teóricos e identificando las alteraciones del trastorno del lenguaje que afecta la articulación de fonemas y que propicia dificultades del habla, por factores como sustitución, distorsión, inserción y omisión de sonidos. El trabajo investigativo tiene un enfoque cualitativo, de tipo explicativo, el mismo que permitió conocer las causas de la dislalia y sus consecuencias. La población a la cual se aplicó el test fue 44 niños, 12 de ellos presentaron trastorno del habla el 19\%, a quienes se aplicó re-test. El resultado fue mejoramiento en la pronunciación de fonemas del 8,3\% de disminución de la afectación: distorsión 0,3\%, inserción 1,8\%, omisión 2\% y sustitución 4,2\%. Lo logrado sustenta que las dislalias son alteraciones de la articulación de fonemas y un defecto de pronunciación, las mismas que se mejoran al intervenir con la realización de ejercicios del paladar, mandíbula, labios y lengua, logrando así, la reducción de las afectaciones en la articulación de fonemas y palabras.
\end{abstract}

Palabras Clave: fonemas, articulaciones de fonemas, tipos de dislalias, trastornos de lenguaje.

Recibido: 21 de julio de 2020

Aceptado: 14 de diciembre de 2020

Publicado como artículo científico en la Revista de Investigación Talentos VII (2), 66-73 
Abstract: The objective of the research was to determine the strategy that contributes to reducing dyslalia, describing the theoretical foundations and identifying the alterations of the language disorder that affects the articulation of phonemes and that leads to speech difficulties due to factors such as substitution, distortion, insertion and omission of sounds. The investigative work has a qualitative, explanatory approach, the same one that allowed us to know the causes of dyslalia and its consequences. The population to which the test was applied was 44 children, 12 of them presented speech disorder in 19\%, to whom a re-test was applied. The result was an improvement in the pronunciation of phonemes of $8.3 \%$ decrease of the affectation: distortion $0.3 \%$, insertion $1.8 \%$, omission $2 \%$ and substitution $4.2 \%$. What has been achieved supports that dyslalias are alterations in the articulation of phonemes and a pronunciation defect, which are improved when intervening with the performance of exercises of the palate, jaw, lips and tongue, thus achieving the reduction of affectations in the articulation of phonemes and words.

Keywords: phonemes, phoneme articulations, types of dyslalias, language disorders.

\section{INTRODUCCIÓN}

La dislalia es un trastorno del lenguaje oral, que afecta al desarrollo del vocabulario. De las investigaciones realizadas en base a un diagnóstico que permita determinar las estrategias de actuación eficaces.; (Remache, 2015); (Gómez, 2015) y (Andrade, 2015), coinciden que son alteraciones de la articulación de fonemas, que es un defecto de pronunciación del mecanismo de la articulatorio.

Los problemas de articulación (dislalias) consisten en la sustitución anormal, distorsión, inserción u omisión de los sonidos del habla, (Barros de Flores \& Flores, Rochow, s.f); es la articulación defectuosa debida a aprendizaje defectuoso o anormalidad de los órganos externos del habla, (Silva, Silva, 2016); es el trastorno de la articulación de los fonemas por alteraciones funcionales de los órganos periféricos del habla, (Cantos, Vasquez, 2016). Presentan trastornos de la pronunciación (o articulación del habla) que no obedece a patología del sistema nervioso central. (Mateo, Ayala, 2010), son anomalías de la pronunciación, (Azcoga, Dermán, \& Iglesias, 1998)

La dislalia es una perturbación del lenguaje que se caracteriza por la incapacidad de pronunciar bien ciertos fonemas; es decir, colocar los órganos fonatorios en la posición correcta. (Rodríguez, 2015)., es un disturbio de pronunciación no por defecto neurológico sino por daño estructural del aparato articulatorio. Puede ser causada por heridas de labios, lengua, paladar, piso de la boca, lesiones máxilo-faciales, perforaciones del paladar, labio leporino, paladar hendido, tumores de paladar y lengua, (Peña, Uranga, 2018), es una sustitución, alteración u omisión de los fonemas en general, (Mérida \& Fernandez, 2003)

El problema del lenguaje oral consiste en la pronunciación errónea de vocales 
ele; siendo consecuencias de múltiples factores (Correa, 2014). Son alteraciones de las articulaciones de algunos fonemas y defectos visibles de pronunciación, debido a alteraciones del mecanismo articulatorio, fonéticas y fonológicas (Yagual 2013). Para que no se den estos errores se debe tener un control adecuado en la ejecución de los mecanismos del habla; además se deberá saber distinguir y recordar los modelos auditivos y reproducirlos exactamente.

El análisis del problema permitió identificar las causas, de la afectación a las articulaciones y la producción de fonemas. La población interviniente muestra trastornos del habla presentando síntomas de omisión, sustitución, distorsión e inserción de fonemas.

Los estudiantes con estos trastornos muestran retrasos significativos en el desarrollo del leguaje y la comunicación entre pares, esta afectación influye en la adquisición y maduración de los esquemas motrices de los fonemas y pronunciación de palabras. Es necesario reflexionar que, desde el inicio del habla hasta los 4 y 5 años, aparecen defectuosas articulaciones del lenguaje en la pronunciación de fonemas; para evitar la persistencia de estos defectos es conveniente una intervención en la etapa inicial del trastorno. La dislalia trata del trastorno del lenguaje, que es una de las patologías de alteraciones de articulación más común en la infancia, que afecta en el habla, así también en el aprendizaje.

Esta investigación se basa en la necesidad diaria en el aula, que conlleva a dificultades para aprender la forma de procesar mentalmente y volver a crear los sonidos hablados; presentándose problemas al pronunciar los fonemas; esto hace que el niño no distinga los sonidos para determinadas palabras. Los factores que definen las causas de dislalia, que originan problemas de aprendizaje, es la no adquisición de una forma correcta, los patrones de movimiento para producción del habla; lo quiere decir, que el niño presenta insuficiente habilidad motora; esto hace que cometa omisiones, sustituciones $\mathrm{y}$ distorsiones de algunos sonidos de la lengua o tienen dificultad de lenguaje, produciéndose alteraciones en la pronunciación. Otra causa de la limitada compresión o discriminación auditiva, debido a que el niño no puede imitar los sonidos porque no los percibe; en otra situación oye bien, pero analiza o hace una integración inadecuada de los fonemas que escucha.

El propósito de la investigación es determinar y aplicar estrategias para reducir la dislalia en niños del Centro Educación Inicial "Las Mercedes" de Babahoyo, describe los fundamentos teóricos e identificando las alteraciones de lenguaje que afectan la articulación, y que propicia las dificultades del habla. La determinación del proceso para la aplicación de estrategias de intervención es parte de este estudio.

\section{MATERIALES Y MÉTODOS}

El alcance de investigación fue explicativo, con enfoque cualitativo, y mediante la observación participativa se explica la realidad sobre los tipos de errores de la variable dislexia que hay al pronunciar fonemas y palabras $y$ 
de la variable técnica vocal para dar solución del problema. La población interviniente fue de 44 niños, al considerar la totalidad no fue necesario determinar una muestra. Las técnicas aplicadas en la investigación fue el test, la observación, un primer momento de manera espontánea y otro momento repetitivo. El instrumento fue Evaluación de la Discriminación Auditiva de los sonidos del habla, (Laiz, M, 2017); el proceso se fundamenta en lectura de imágenes presentado a los niños en forma individual, el mismo que reconoce y nombra la figura que observa; esta acción permitió detectar el problema. La investigación profundizó los fundamentos epistemológicos, el conocimiento de las afectaciones a la articulación de los fonemas y se propuso estrategias para dar solución al problema del lenguaje y la comunicación. Para el análisis datos se aplicó el test y retest, la instrucción consistió en solicitarle al niño que lean en voz alta la imagen que mira, las respuestas de la lectura fueron asentadas en la hoja de registro los que posterior con el uso del Excel se realizó el análisis. La estrategia de intervención consistió luego de identificar los errores de la aplicación del test; se trabajó con ejercicios del paladar a través de soplar objetos y hacer gárgaras, ejercicios de mandíbula, inflar y desinflar globos de aire, ejercicios con los labios simular un beso y ejercicios con la lengua, sacar y meter la lengua con la boca abierta.

Para el análisis de correlación entre la técnica vocal y las dislalias distorsión, inserción, omisión, sustitución y sin error en los dos momentos, con aplicación del estadístico Pearson, utilizando el programa Excel, tras esta aplicación arroja el valor de 0,957, el mismo que es significativo, $y$ un $\mathrm{p}$ valor de 0,043 . Con $p$-valor $<0,05$, se acepta que la pronunciación de imágenes ayuda a reducir la afectación de las deficiencias de articulación para la pronunciación de palabras.

Para la aplicación del instrumento se socializó a los representantes legales el objetivo de la investigación, quienes dieron la anuencia aspecto ético del proceso.

Tabla 1. Registro de errores de pronunciación de fonemas observados en los niños

\begin{tabular}{|c|c|c|c|c|c|c|c|}
\hline & Items & 趈 & 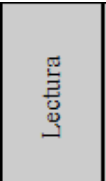 & 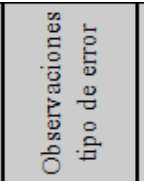 & 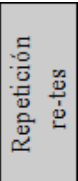 & 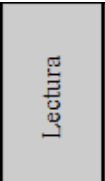 & 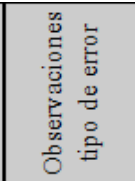 \\
\hline$/ \mathrm{p}-1$ & Palo & + & palo & sin error & + & palo & sin error \\
\hline$/-\mathrm{p}-1$ & mapa & - & apa & omisión & - & apa & omisión \\
\hline$/ \mathrm{m} /$ & mama & + & mama & sin error & + & mama & sin error \\
\hline $\mathrm{k}-1$ & cama & - & ama & omisión & - & ama & omisión \\
\hline$/-\mathrm{k}-/$ & Poco & - & oco & omisión & - & oco & omisión \\
\hline$/ b-/$ & Bata & - & ata & omisión & + & Bata & sin error \\
\hline -b- & bebé & + & bebe & sin error & + & bebe & sin error \\
\hline$/ g-1$ & Gato & - & lato & sustitución & $=$ & lato & sustitución \\
\hline $\mid-\mathrm{g}-1$ & mago & + & mago & sin error & + & \begin{tabular}{|l|} 
mago \\
\end{tabular} & sin error \\
\hline$/ \mathrm{x}-1$ & jirafa & - & jsifara & inserción & - & jsifara & inserción \\
\hline$|-x-|$ & Ojo & - & $\infty$ & distorsión & - & 00 & distorsión \\
\hline$/ \mathrm{f}-/$ & Foca & - & oca & omisión & - & oca & omisión \\
\hline$|-\mathrm{f}-|$ & café & - & $\overline{a f e}$ & omisión & - & afe & omisión \\
\hline$/ z-1$ & zapato & - & ssapato & inserción & - & ssapato & inserción \\
\hline$|-z-|$ & Taza & - & asa & Inserción & - & asa & Inserción \\
\hline$/ \mathrm{s}-1$ & Seta & - & zeta & sustitución & - & zeta & sustitución \\
\hline$|-\mathrm{s}-|$ & Vaso & + & vaso & sin error & + & vaso & sin error \\
\hline t -1 & Toro & - & Ioro & sustitución & - & Ioro & sustitución \\
\hline$|-t-|$ & moto & + & moto & sin error & + & moto & sin error \\
\hline$/ \mathrm{d}-/$ & dominó & + & domino & sin error & + & domino & sin error \\
\hline$|-1-|$ & helado & - & lelado & sustitución & - & lelado & sustitución \\
\hline $1-1$ & lupa & - & upa & omisión & - & upa & omisión \\
\hline $\mid-1-/$ & pela & - & tela & sustitución & $=$ & tela & sustitución \\
\hline$/ \mathrm{n}-1$ & Niño & + & niño & sin error & + & niño & sin error \\
\hline$/-\mathrm{n}-/$ & mano & + & mano & sin error & + & mano & sin error \\
\hline$|-r-|$ & maraca & + & ramaca & sin error & + & ramaca & sin error \\
\hline$/ \mathrm{r}-1$ & rata & - & lata & sustitución & - & lata & sustitución \\
\hline$|-r-|$ & torre & - & Iorre & sustitución & $=$ & Iorre & sustitución \\
\hline$/ \mathrm{c}-1$ & chino & + & chino & sin error & + & chino & sin error \\
\hline$|-c-|$ & cuchara & - & tuchara & sustitución & - & tuchara & sustitución \\
\hline $1-1$ & llama & - & dama & sustitución & - & \begin{tabular}{|l|} 
dama \\
\end{tabular} & sustitución \\
\hline $\mid-1-1$ & medalla & + & medalla & sin error & + & Medalla & sin error \\
\hline $\mid-n-/$ & muñeco & - & luñeco & sustitución & - & luñeco & sustitución \\
\hline
\end{tabular}

Fuente: Elaboración propia de los autores: septiembre 2018 
Interpretación: De la aplicación del instrumento se observa que en 22 palabras los niños presentan dislalia en la pronunciación de fonemas; en 1 palabra error de distorsión de sonidos; en 4 palabras con error de inserción al añadir en la pronunciación sonidos que no corresponden a la palabra; en 7 palabras error de omisión de fonemas de la palabra y en 10 palabras error de sustitución de un fonema que no puede pronunciar en forma correcta por otro. El registro de la observación posterior a la intervención muestra una reducción en el tipo de error es de omisión y sustitución.

\section{RESULTADOS Y DISCUSIÓN}

Tabla 2. Errores en la pronunciación de fonemas observados en los niños en la aplicación del test, que visibilizan la dislalia

\begin{tabular}{lcccccccccccccc}
\hline $\begin{array}{l}\text { Error/Su- } \\
\text { jetos }\end{array}$ & 1 & 2 & 3 & 4 & 5 & 6 & 7 & 8 & 9 & 10 & 11 & 12 & Suma & $\%$ \\
\hline Distorsión & 1 & 1 & 1 & 1 & 1 & 1 & 1 & 1 & 1 & 1 & 1 & 1 & 12 & $3 \%$ \\
Inserción & 7 & 3 & 3 & 3 & 4 & 4 & 4 & 4 & 4 & 4 & 4 & 4 & 48 & $12 \%$ \\
$\begin{array}{l}\text { Omisión } \\
\begin{array}{l}\text { Sustitu- } \\
\text { ción }\end{array}\end{array}$ & 3 & 7 & 7 & 7 & 7 & 5 & 6 & 7 & 7 & 7 & 7 & 7 & 77 & $19 \%$ \\
Sin error & 10 & 10 & 11 & 10 & 10 & 11 & 10 & 11 & 11 & 9 & 9 & 10 & 122 & $31 \%$ \\
Total & 33 & 33 & 33 & 33 & 33 & 33 & 33 & 33 & 33 & 33 & 33 & 33 & 396 & $100 \%$ \\
\hline
\end{tabular}

Fuente: Elaboración propia de los autores; septiembre del 2018

De la aplicación del instrumento previa a la intervención se observa que el $67 \%$ de palabras los niños presentan dislalia en la pronunciación de fonemas presentan, el $3 \%$ errores de distorsión de sonidos; el $12 \%$ errores de inserción al añadir en la pronunciación sonidos que no corresponden a la palabra; el $21 \%$ errores de omisión de fonemas de la palabra y el $0 \%$ errores de sustitución de un fonema que no puede pronunciar en forma correcta por otro; estos registros muestra la incidencia y presencia de dislalia en los niños.

Tabla 3. Errores de pronunciación fonemas observados en los niños en la aplicación del re-test, con los que se visibiliza la incidencia de la intervención en la reducción de la dislalia

\begin{tabular}{lcccccccccccccr}
\hline Sujetos & 1 & 2 & 3 & 4 & 5 & 6 & 7 & 8 & 9 & 10 & 11 & 12 & Suma & \multicolumn{1}{c}{$\%$} \\
\hline Distorsión & 1 & 1 & 1 & 1 & 0 & 1 & 1 & 1 & 1 & 1 & 1 & 1 & 11 & $3 \%$ \\
Inserción & 3 & 3 & 3 & 3 & 4 & 4 & 4 & 3 & 3 & 4 & 3 & 4 & 41 & $10 \%$ \\
Omisión & 6 & 6 & 6 & 6 & 5 & 5 & 5 & 6 & 5 & 7 & 6 & 6 & 69 & $17 \%$ \\
sustitución & 10 & 8 & 9 & 8 & 9 & 10 & 8 & 10 & 10 & 7 & 8 & 8 & 105 & $27 \%$ \\
sin error & 13 & 15 & 14 & 15 & 15 & 13 & 15 & 13 & 14 & 14 & 15 & 14 & 170 & $43 \%$ \\
$\quad$ Total & 33 & 33 & 33 & 33 & 33 & 33 & 33 & 33 & 33 & 33 & 33 & 33 & 396 & $100 \%$ \\
\hline
\end{tabular}

Fuente: Elaboración propia de los autores; septiembre del 2018 
De la repetición de la aplicación del instrumento previa a la intervención se observa que en 19 palabras los niños presentan dislalia en la pronunciación de fonemas presentan los registro observados posterior a la intervención muestran prevalencia 1 palabra en distorsión,
4 palabras en inserción, 6 palabras con omisión y 8 palabras con sustitución; Esto demuestra que, con la aplicación del plan de intervención durante 15 días, se presentó una reducción de dislalia en el 8\%.

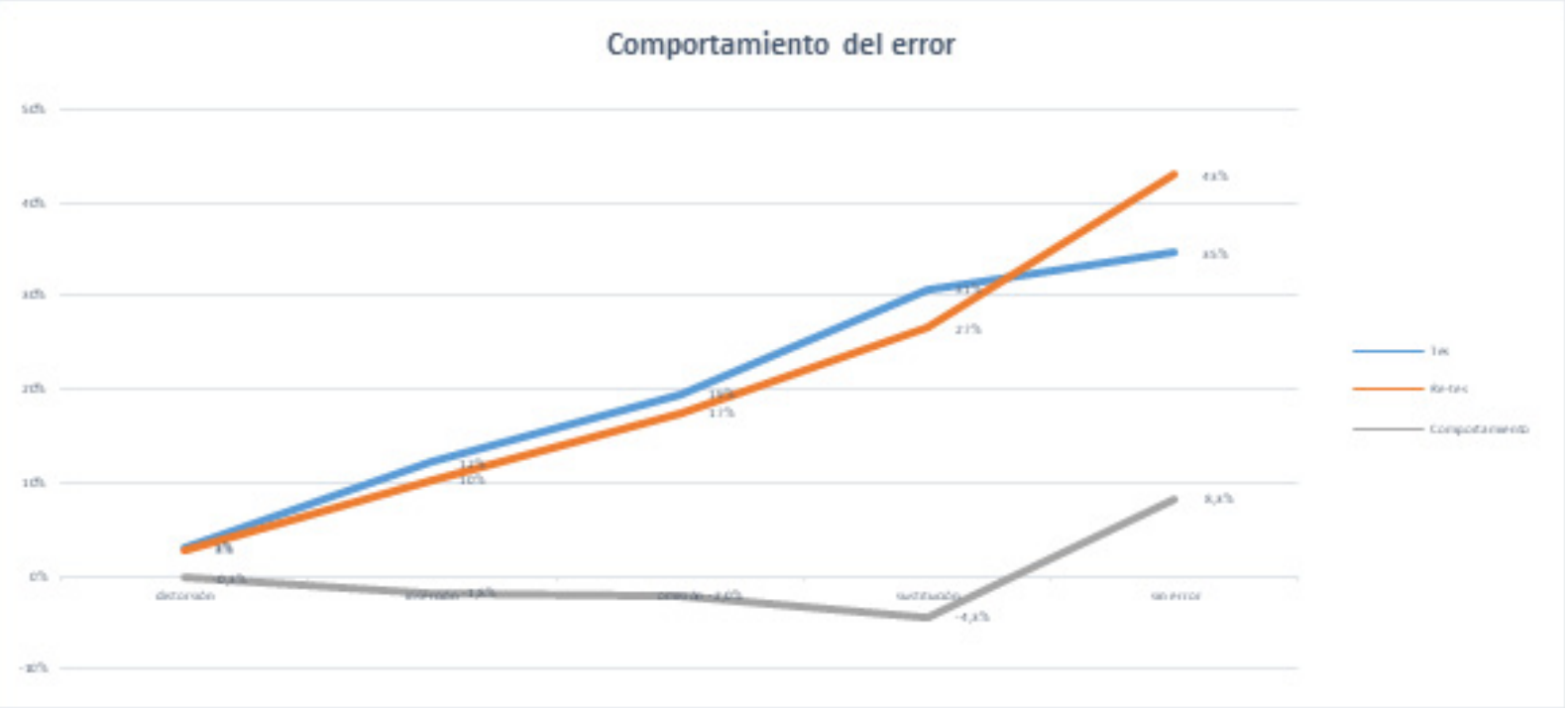

Figura 1. Analís del comportamiento prevalencia de los errores de la dislalia, con la aplicación de la prueba (test), antes y después de la intervención

La implementación de estrategias de intervención permitió alcanzar el objetivo, el que fue el de reducir los errores de dislalia en los niños, con base a los datos de la aplicación de test y re-test, se logró mejorar la articulación que afecta al habla, se observa una reducción global de los trastornos del habla en $8,3 \%$, visibilizándose: distorsión $-0,3 \%$, inserción $-1,8 \%$, omisión $-2 \%$, sustitución $-4,3 \%$. En síntesis, existe mejoramiento en la articulación de fonemas y se determina que una intervención fonética, logra la pronunciación correcta de fonemas y mejora las funciones que inciden en la expresión oral, trabajando las bases funcionales entre el desarrollo de habilidades motoras de los órganos de articulación y de respiración.
Elgrupo interiorintervenido, con antecedentes del 57\% de dificultades en la articulación de fonemas y palabras al nombrar las imágenes contempladas en el instrumento aplicado, demuestra un cambio en el valor y porcentaje de afectación de la articulación de fonemas, pasando el aspecto sin error del 35\% al 43\%, esto evidencia los beneficios de establecer una estrategia de intervención para reducir las afectaciones con lo que se mejora el habla y lenguajes.

Los investigadores del presente estudio, coinciden con los autores citados en la investigación, en que las dislalias son alteraciones de la articulación de fonemas, un defecto de pronunciación del mecanismo de la 
articulatorio, compartiendo los investigadores este criterio, y que es necesario puntualizar que el docente o la familia, pueden intervenir en la posibilidad de corregir la dislalia fonológica y fonética, estableciendo acciones para reducir factores como la sobreprotección de los padres, y además consolidar en el aula a través de planificaciones diferenciadas.

Así mismo se coincide con lo que se menciona sobre el momento de clasificar los tipos de dislalia, se evidencia que existe además la dislalia evolutiva, en el que el niño no repite por imitación lo que escucha; Dislalia audiógena produce por insuficiente audición que afecta al proceso de maduración de la personalidad; dislalia orgánica, por trastornos de articulación a causa de alteraciones orgánicas referidas al sistema nervioso; dislalia funcional, generada por mal funcionamiento o lesión de los órganos de articulación, causante de una deficiente coordinación musculary la dislalia fonológica alteración de los sonidos que conforman la palabra; dislalia fonológica alteración a nivel perceptivo y organizativo en los mecanismos de programación, con la producción de palabras donde se visibiliza los errores de omisión, sustitución, inserción y distorsión. Estas dificultades de articulación que hacen difícil el habla provocan disminución en las relaciones sociales, presentándose con mayor frecuencia, conductas des adaptivas.

\section{CONCLUSIONES}

La investigación determina que la técnica vocal a través de lectura de imágenes es una estrategia que contribuye a reducir en el 8,3\% las afectaciones a la articulación de fonemas y palabras. La solución fundamental es aplicar la técnica vocal, la cual mejora el desarrollo de la comunicación, supera las dificultades de articulación o para una producción clara y fluida de la voz, ayudando a la inteligibilidad y a la expresión oral tanto en el ámbito personal y social

La afectación del trastorno del lenguaje por deficiencias de articulación de fonemas es dos de diez del grupo de niños que asisten al nivel inicial en la institución intervenida. La identificación y prevalencia de afectación a las alteraciones del lenguaje del 19\% de errores; permita establecer acciones y el establecimiento de medidas correctivas a través de una estrategia de intervención pedagógica.

La intervención pedagógica debe centrarse en la dislalia funcional, ya que esta es notoria debido a la no articulación de fonemas la /r/, /s/, /x/, /m/, /1/, /n/, /ñn/, /k/, /f/, /g/, /ch/, /p/, /t/, /11/, pronunciación de la /s/ por /z/, o/z/ por /s/, o por la sustitución de la /r/ por /1/, la /s/ por la /ch/; dificultad de articulación que hacen difícil el habla.

\section{REFERENCIAS BIBLIOGRÁFICAS}

Andrade; Gualacata, P. R. (2015). Movimientos faciales y su incidencia en el desarrollo del lenguaje oral de los niños y niñas de 3-4 años. Movimientos faciales y su incidencia en el desarrollo del lenguaje oral de los niños y niñas de 3-4 años de la Unidad Educativa 28 de Abril de la 
ciudad de Ibarra, provincia de Imbabura durante el periodo escolar 2014- 2015. Ibarra, Imbabura, Ecuador.

Azcoga, J., Dermán, B., \& Iglesias, A. (1998). Alteraciones del Aprendizaje Escolar. Paidos Iberica, Ediciones S. A.

Barros de Flores, A., \& Flores, Rochow, F. (s.f). La dislalia: Problema del lenguaje o problema del habla. Scielo.conicyt.

Cantos, Vasquez, E. D. (2016). Aplicación de ejercicios de discriminación auditiva y segmentación de palabras favoreciendo la conciencia fonológica en niños con dislalia funcional de 4 a 6 años. Guayaquil, Guayas, Ecuador.

Correa; Chávez, C. M. (2014). Aplicación de un programa de intervención psicopedagógica en problemas de articulación de fonemas. Aplicación de un programa de intervención psicopedagógica en problemas de articulación de fonemas, para mejorar el desarrollo del lenguaje oral en los estudiantes del segundo grado de educación primaria en el área de comunicación de la institución educati. La Libertyad, Santa Elena, Ecuador.

Gómez, Cordero, C. V. (2015). Aplicativo Android para controlar trastornos de articulación de los fonemas denominado dislalia en menores entre 1 y 5 años. Guayaquil, Guayas, Ecuador.

Mateo, Ayala, E. J. (2010). Los antecedentes de la eximente de anomalía o alteración psíquica. Dinkinson.

Mérida, C., \& Fernandez, Y. (2003). Intervención logopédica en los trastornos fonológicos desde el paradigma psicolingüístico del procesamiento del habla. Neurol.

Peña, Uranga, I. (2018). Propuesta de intervención para un niño con dislalias funcionales. Valladolid, España.

Remache, Pillagua, M. D. (enero de 2015). La dislalia y su impacto en el desarrollo socioemocional en los niños de 5 a 6 años. La dislalia y su impacto en el desarrollo socioemocional, en los niños de 5 a 6 años: Diseño de un programa de capacitación para representantes legales. Guayaquil, Guayas, Ecuador.

Rodríguez, J. O. (25 de 09 de 2015). Estrategias didácticas para mejorar el rendimiento de los estudiantes de educación regular con dificultades de dislalia en la Escuela Fiscal Matutina Ayúdanos a Empezar del cantón Babahoyo, provincia de los Ríos. Babahoyo, Los Ríos, Ecuador.

Silva, Silva, M. H. (2016). Dislalia y su repercusión en la articulación de palabras a estudiantes de la Escuela de Educación Básica "Juan León Mera", cantón Montalvo, provincia Los Ríos. Babahoyo.

Yagual, Bazán, H. M. (2013). Manual con ejercicios articulatorios. Manual con ejercicios articulatorios para la atención de la dislalia funcional de los niños y niñas de 5 años del centro de educación básica fiscal $n^{\circ} 2$ general César Rohón Sandoval de la parroquia Anconcito del cantón salinas, provincia de Santa Elena, d. Santa Elena, Santa Elena, Ecuador. 